\title{
Efecto del entrenamiento pliométrico en la fuerza explosiva de niñas puberes practicantes de voleibol \\ Effects of plyometric training on explosive strength in pubescent girls volleyball players
}

\author{
*Gustavo Vilela, *,**Alexis Caniuqueo-Vargas, ***Rodrigo Ramirez-Campillo, ***, *****Claudio \\ Hernández-M osqueira, *Sandro Fernandes da Silva \\ *U niversidad Federal de Lavras (Brasil), **Universidad Católica deTemuco (Chile), ***U niversidad de Los Lagos (Chile), \\ $* * * *$ Universidad Adventista de Chile (Chile)
}

Resumen. El objetivo del estudio fue evaluar los efectos de un programa de entrenamiento pliométrico en la potencia muscular de niñas practicantes de voleibol ( $n=78$; edad $=12,18 \pm 1,27$ años). Las participantes fueron divididas subdividas de acuerdo con el grado de maduración en: grupo control ( $G C=48$ con grado de maduración $2=11 ; 3=19 ; 4=19)$ y grupo de trabajo (GT $=30$ con grado de maduración $2=10 ; 3=10 ; 4=10$ ). Ambosgruposreal izaron 3 sesiones semanales de entrenamiento devoleibol, adicionando 3 sesiones de ejercicios pliométricosaGT queincluyeron salto en zigzag, linealesy profundidad. Para la eval uación pre y post entrenamiento se utilizó test Squat Jump, Saltos M ovimiento, Drop Jump y A bal akov. La estadística prueba t student para significancia pre y post entrenamiento $(p<0,05)$ y también el análisis de la magnitud del efecto. Los resultados no reportar on diferencias significativas post entrenamiento a pesar de registrar pequeños y moderadas magnitudes de efecto. Se concluye que el entrenamiento polimétrico no genero efectos positivos en la saltabilidad de niñas púberes que practican voleibol.

Palabras Clave: Entrenamiento, Pubertad, Ejercicio Pliométrico, Voleibol.

\begin{abstract}
The aim of this study was to eval uate the effects of plyometric training (PT) on muscle power (PR) of the lower limbs in girls volleyball players aged ( $n=78 ; 12.18 \pm 1.27$ years). Participants were divided according to the degree of maturation into control group (GC $=48$ with maturity level $2=11 ; 3=19 ; 4=19$ ) and working group (GT $=30$ with maturity degree $2=10 ; 3=10 ; 4=10$ ). held three sessions per week of volleyball and experimental group - EG $n=24$, which held in addition of 3 weekly sessions of volleyball, more 3 weekly sessions of PT that comprised the multi jump, jump over hurdle and drop jumps. For pre- and post-training eval uation test was used Squat Jump, Counter M oviment Jump and Jump D rop A balakov. The statistical student t test for significance Pre-post $(p<0.05)$ and the analysis of the magnitude of the effect. The results reported no significant differencestraining post despite posting small to moder ate effect sizes. It is concluded that the polymetric training did not generate positive effects on the saltability of pubertal girls who practice volleyball.
\end{abstract}

Key Words: Training, Puberty, Plyometric Exercise, Volleyball.

\section{Introdución}

Las condiciones de sal tabilidad y fuerza explosiva son considerados factores fundamentales en el rendimiento de deportes que requieren altas demandas de potencia y velocidad (Lebedew et al., 2016; Sánchez-M oreno, García-Asencio, González-Badillo, \& Díaz-Cueli, 2018) - Por ello, los métodos de entrenamientos basados en pliometría, han constituido un medio eficaz para el desarrollo de fuerza explosiva, velocidad y saltabilidad en deportes como voleibol, atletismo, básquetbol y futbol (Markovic, 2007; Martínez-rodríguez, Mira-alcaraz, Cuestas-calero, Pérez-turpín, \& Pedro, 2017; Saez de Villarreal, E, Suarez-Arrones, L, Requena, B, H aff, GG,

Fecha recepción: 28-01-20. Fecha de aceptación: 08-10-20 Sandro Fernandes da Silva sandrofs@ufla.br
Ferrete, 2015; Sánchez-Sixto \& Floría, 2016).

En el caso del voleibol, ha sido reconocido como deporte de velocidad, explosivo, de alta precisión técnica (G Battaglia, A Paoli, M Bellafiore, A Bianco, 2014; Kotzamanidis, 2003; M artinez, Mira, \& Cuestas, 2017), que requiere de entrenamientos que optimicen la función fisiológica para movimientos de alta potencia a diferentes velocidades (Pereira, Costa, Santos, Figueiredo, \& J oão, 2015; Sánchez-M oreno et al. , 2018), siendo varios los investigadores que han reportado estas características en jugadores de esta especialidad (K abacinski et al., 2016; Kipp, Kiely, \& Geiser, 2016; Vilela, G; Da Silva, 2017).

Diversos investigadores han reportado efectos positivos del entrenamiento polimétrico sobre la coordinación intramuscular, mejoras en la sincronización de la actividad muscular y de la actividad mitótica, aumento de la eficiencia neural, mejoras propioceptivas y con- 
trol neuromuscular (Dariusz M roczek, Krzysztof M aækaBa, Adam Kawczynski, Edward Superlak, PaweB Chmura, Tomasz Seweryniak, 2018; Seitz \& H aff, 2016; Stojanoviæ, Ristiæ, M cM aster, \& M ilanoviæ, 2017;Tillin, Pain, \& Folland, 2013), además de mejoras en el rendimiento de saltabilidad, fuerza explosiva, resistencia y balance (Gjinovci, Idrizovic, Uljevic, \& Sekulic, 2017a; Ramírez-Campillo et al., 2015; Rodrigo Ramírez-Campillo, César M eylan, CristianAlvarez, Carlos HenríquezO Iguín, Cristian Martínez, Rodrigo Cañas-Jamett, Da vid C Andrade, 2014). También, ha sido señalado un método apropiado de rehabilitación y prevención de lesiones por los efectos positivos en el control motor en cualquier nivel y edad (J ohnson, Salzberg, \& Stevenson, 2011; Jorge R Fernandez-Santos, Jonatan R Ruiz, Da niel D Cohen, Jose L Gonzalez-M ontesinos, 2015; M CKay et al., 2005; Rafael Martin Acero, Miguel Fernándezdel O Imo, JoseA ndres Sánchez, X ose LuisO tero, Xavier Aguado, 2011; Sattler et al., 2018).

Respecto al entrenamiento pliométrico en niño y jóvenes, siempre debe ser destacado es la importancia de conocer la madurez de jóvenes, ya que la misma puede representar un mejor rendimiento físico/ fisiológico, ya que las condiciones hormonales y neuronales van interferir en esas respuestas(Beltran-Valls, Adelantado-Renau, Segura-Ayala, Toledo-Bonifás, \& MolinerU rdiales, 2019). Investigaciones ya aportan efectos positivos en la salud, postura y rendimiento, enfatizando en la responsabilidad y supervisión de la aplicación de estos programas en sujetos jóvenes (A nis Zribi, M ohamed Zouch, Hamada Chaari, Elyes Bouajina, Hela Ben N asr, M onia Zaouali, 2014; Chaouachi et al. , 2012; Johnson et al., 2011; Kotzamanidis, 2003). A pesar de ello, las investigaciones respecto a los efectos de este tipo de entrenamiento en jóvenes que practican voleibol son de escases en la literatura, más aún, aquellos que estudien los efectos a partir de la maduración biológica, por ello, el objetivo para los investigadores fue estudiar los efectos del entrenamiento pliométrico en la fuerza explosiva de niños en estado de desarrollo puberal.

\section{Métodos}

\section{Enfoque del estudio}

El estudio contemplo un programa de entrenamiento pliométrico de 8 semanas, divididos en tres sesiones semanales, días alternados y posterior al entrenamiento de voleibol. El entrenamiento incluyo saltos en dos pies, salto con cambio hacia anterior, salto zig zag, salto de vallas con dos piernas y salto profundo de cajón. Las participantes fueron asignados al azar, y subdividos de acuerdo con el grado de maduración en: grupo control ( $G C=48$ con grado de maduración $2=11 ; 3=19 ; 4=19$ ) y grupo de trabajo ( $\mathrm{GT}=30$ con grado de maduración 2=10; 3=10; 4=10). La maduración sexual fue basada en las características sexuales secundarias deTanner frente a la auto inspección visual y comparaciones con la referencia normativa fotográfica.

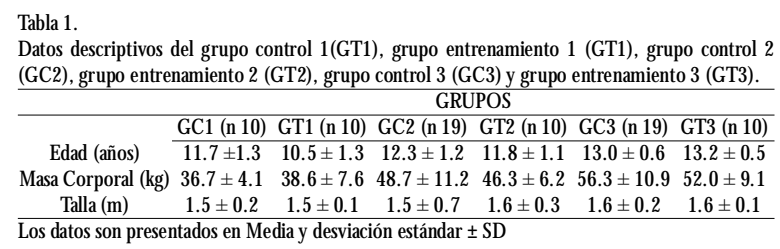

Datos descriptivos del grupo control 1(GT1), grupo entrenamiento 1 (GT1), grupo control 2 $\frac{\text { (GC2), grupo entrenamiento } 2 \text { (GT2), grupo control } 3(\mathrm{GC3}) \text { y grupo entrenamiento } 3 \text { (GT3). }}{\text { GRUPOS }}$

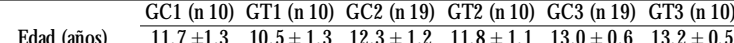
M asa Corporal $(\mathrm{kg}) \quad 36.7 \pm 4.1 \quad 38.6 \pm 7.6 \quad 48.7 \pm 11.2 \quad 46.3 \pm 6.2 \quad 56.3 \pm 10.9 \quad 52.0 \pm 9.1$ \begin{tabular}{lllllll} 
Talla $(\mathrm{m})$ & $1.5 \pm 0.2$ & $1.5 \pm 0.1$ & $1.5 \pm 0.7$ & $1.6 \pm 0.3$ & $1.6 \pm 0.2$ & $1.6 \pm 0.1$ \\
\hline
\end{tabular} Los datos son presentados en Media y desviación estándar \pm SD

\section{Muestra}

Se reclutaron 78 niñas, púberes, con edad 12,3 \pm 1,2 años, peso 48,2 $\pm 11,3 \mathrm{~kg}$, talla 1,60 $\pm 1,1 \mathrm{~m}$, voluntarias del sexo femenino, practicantes de voleibol de tres escuelas deportivas de la ciudad de Lavras, Minas Gerais, Brasil. Los criterios de inclusión considera ron practicar voleibol por un período mínimo de seis meses y edad entre 9 y 14 años. Se excluyeron a aquellos sujetos que no participaron de todas las sesiones de entrenamiento Pliométrico 0 entrenaron otros ejercicios físicos en paralelo, en que así fueron excluidas del estudio, niñas del GT.

El proyecto fue enviado y aprobado por el Comité de Ética de la Universidad Federal de Lavras - MG Brasil, con el no CAAE 03214312.5.0000.5148. Lospa dres firmaron el Consentimiento Informal con informaciones sobre la participación de su hija, conociendo los detalles den entrenamiento y evaluaciones e informando el carácter experimental de esta investigación, conforme resolución CNS no 196/ 96.

\section{Procedimientos}

Las evaluaciones fueron realizadas pre y post entrenamiento, durante el periodo del estudio no hubo partidos, siendo caracterizado como un periodo de prepa ración de los equipos. Los intervalos entre las evalua ciones fueron de 4 semanas. Para evaluación de la fuerza explosiva de los miembros inferiores se utilizó la Plataforma) umptest $\circledast(100 \times 66 \mathrm{~cm})$ delamarca Hidrofit, conectado el software Jump Test. 2.0, todos los testes fueron en el periodo de la tarde entre las 18:00-19:00 horas. Se consideró los test de saltos Squat Jump (SI) salto sin contra movimiento previo, realizado empezando de una flexión de rodillas en $90^{\circ}$, con las manos en la cintura; Counter M oviment Jump (CMJ) salto con 
diferencias pre y post entrenamiento no sean significa tivas, contrario a lo reportado (Pereira et al., 2015; Sattler et al., 2018), en jugadoras de voleibol durante 8 y 12 semanas de entrenamiento pliométrico, concluyendo los investigadores que periodos de descanso podrían generar efectos significativos en las mejoras de la fuerza explosiva. En este sentido, el presente estudios pese a utilizar mismos interval os no evidencia resulta dos similares, pudiendo ser condicionada por la edad y sexo, sin embargo, en otros estudios (Branislav, Milivoj, Abella, Deval, \& Siniša, 2013; Kabacinski et al., 2016; Kotzamanidis, 2003; Marques, M.A.C.; GanzálezBadillo, 2006; Poulos et al., 2018; Sattler et al., 2018), que comparan los efectos en función del sexo, se registraron efectos significativos para ambos grupos, concluyendo los investigadores que las adaptaciones al entrenamiento polimétrico no difieren entre hombres y mujeres(Martínez-rodríguez et al., 2017), además de poner en evidencia de planear las transferencias del entrenamiento pliométrico hasta la evaluación del salto vertical (Frayne, Zettel, Beach, \& Brown, 2020; M oran et al., 2020).

0 tro estudio desarrollado que combinaba entrena miento de pliometría y velocidad, reporto resultados positivos en CMJ y Abalakov (Idrizovic et al., 2018; Kotzamanidis, 2003), recomendado la inclusión de este tipo de entrenamiento durante la pubertad reportando la importancia de llevar en consideración la madurez(Beltran-Valls et al., 2019; Gjinovci et al., 2017a). Resultados similares se han reportado por en estudios que han combinado el entrenamiento con saltosy velocidades (Gjinovci, Idrizovic, Uljevic, \& Sekulic, 2017b; Kristof Kipp, Michael T Kiely, 2016; Poulos et al., 2018; Rafæel Martin Acero, Miguel Fernández-del O Imo, Jose Andres Sánchez, Xose Luis O tero, Xavier Aguado, 2011; Sánchez-Sixto \& Floría, 2016).

En el caso del voleibol, se han reportado efectos positivos por entrenamiento pliométrico en distintas semanas, tanto en la saltabilidad como en la fuerza explosiva (Berton, Lixandrão, Pinto eSilva, \&Tricoli, 2018; Pereira et al., 2015; Sattler et al., 2018; Ziv \& Lidor, 2010), en cuanto otro estudio realizo 6 semanas de entrenamiento pliométrico en agua en jugadores de voleibol femenino (15 \pm 1 años) reporto mejoras significativas en la saltabilidad (M cKay et al., 2005), resaltando que el trabajo en el agua puede ayudar en la prevención de lesiones y adaptación de jóvenes jugadoras(McK ay et al., 2005; Soylu, Altundad, Akarçe-me, \& Yildirim, 2020).

\section{Conclusiones}

A partir de lo anterior, se concluye que el entrena miento pliométrico puede traer efectos positivos en las deportistas de voleibol en maduración puberal, pese a no identificar diferencias significativas posteriores en un programa de entrenamiento pliométrico. La maduración biológica podría ser un factor que considerar al momento de estudiar los efectos del entrenamiento, los que, en etapa puberal, podrían ser menores que los reportados en otros estudios en varones.

\section{Referencias}

Anis Zribi, M ohamed Zouch, Hamada Chaari, Elyes Bouajina, HelaBen N asr, M oniaZaouali, Z.T. (2014). Short-term lower-body plyometric training improves whole body BMC, bone metabolic markers, and physical fitness in early pubertal male basketball players. Pediatric ExerciseScience, 26(1), 2232. https: / / doi.org/ 10.1123/ pes. 2013-0053

Beltran-Valls, M. R., Adelantado-Renau, M., SeguraAyala, D., Toledo-Bonifás, M., \& Moliner-U rdiales, D. (2019). M aturational development, physical activity, and sleep quality in adolescent girls: DADOS project. Retos, 2041(35), 71- 74.

Berton, R., Lixandrão, M. E., Pinto e Silva, C. M., \& Tricoli, V. (2018). Effects of weightlifting exercise, traditional resistance and plyometric training on countermovement jump performance: a metaanalysis. Journal of Sports Sciences, 36(18), 2038- 2044. https: / / doi.org/ 10.1080/ 02640414.2018.1434746 Chaouachi, A., Turki-Belkhiria, L., Chtourou, H., Chtara, M., Amri, M., Behm, D. G., ... Turki, 0. (2012). Eight weeks of dynamic stretching during warm-ups improves jump power but not repeated or singlesprint performance. European Journal of Sport Science, 14(1), 19-27. https:/ / doi.org/ 10.1080/ 17461391.2012.726651

Dariusz Mroczek, Krzysztof MaækaBa, Adam Kawczynski, Edward Superlak, PaweB Chmura, Tomasz Seweryniak, J. C. (2018). Effects of volleyball plyometric intervention program on vertical jumping ability in male volleyball players. The Journal of Sports M edicine and Physical Fitness, 58(11), 16111617.

Frayne, D. H., Zettel, J. L., Beach, T. A. C., \& Brown, S. H. M. (2020). The Influence of Countermovements on Inter-Segmental Coordination and Mechanical Energy Transfer during Vertical Jumping Journal of 
Motor Behavior, 0(0), 1-13. https:/ / doi.org/ 10.1080/00222895.2020.1810611

G Battaglia, A Paoli, M Bellafiore, A Bianco, A. P. (2014). Influence of a sport-specific training background on vertical jumping and throwing performance in young female basketball and volleyball players. The Journal of Sports M edicine and Physical Fitness, 54 (5), 581-587.

Gjinovci, B., Idrizovic, K., Uljevic, 0., \& Sekulic, D. (2017a). Plyometric training improves sprinting, jumping and throwing capacities of high level female volleyball players better than skill-based conditioning. Journal of Sports Science and M edicine, 16(4), 527-535.

Gjinovci, B., Idrizovic, K., Uljevic, 0., \& Sekulic, D. (2017b). Plyometric training improves sprinting, jumping and throwing capacities of high level female volleyball players better than skill-based conditioning. Journal of Sports Scienceand M edicine, 16(4), 527-535. Hopkins, W. G., Marshall, S. W., Batterham, A. M., \& Hanin, J. (2009, January). Progressive statistics for studies in sports medicine and exercise science. Me dicine and Science in Sports and Exercise. https:/ / doi.org/ 10.1249/ MSS. 0b013e31818cb278

Idrizovic, K., Sekulic, D., Uljevic, 0., Spasic, M., Gjinovci, B. , João, P. V., \& Sattler, T. (2018). The effects of 3-month skill-based and plyometric conditioning on fitness parameters in junior female volleyball players. Pediatric ExerciseScience, 30 (3), 353363. https:/ / doi. org/ 10.1123/ pes. 2017-0178

Jaroslae Kabacinski, Lecholslaw B Dworak, Michal Murawa, John 0 starello, Agata Rzepnicka, J. M. (2016). A comparison of take-off dynamics during three different spikes, block and counter-movement jump in female volleyball players. Journal of Sports M edicine and Physical Fitness, 56 (12), 1482-1487.

Johnson, B. A., Salzberg, C. L., \& Stevenson, D. A. (2011). A systematic review: Plyometric training programs for young children. Journal of Strength and Conditioning Research, 25(9), 2623-2633. https:// doi.org/ 10.1519/ JSC.0b013e318204caa0

Jorge R Fernandez-Santos, Jonatan R Ruiz, Daniel D Cohen, Jose L Gonzalez-M ontesinos, J. C. -P. (2015). Reliability andValidity ofTests to Assess Lower-Body Muscular Power in Children. Journal of Strength and Conditioning Research, 29(8), 2277-2285. https:/ / doi.org/ 10.1519/ JSC.0000000000000864

Kotzamanidis, C. (2003). The effect of sprint training on running performance and vertical jumping in preadolescent boys. Journal of H uman M ovement Studies, 20 (2), 441-445. https:/ / doi. org/ refwid:7395

Kristof Kipp, Michael T Kiely, C. F. G. (2016). Reactive
Strength Index Modified Is a Valid Measure of Explosiveness in Collegiate Female Volleyball Players. Journal of Strength and Conditioning Research, 30 (5), 1341- 1347. https:/ / doi.org/ 10.1519/ JSC. 0000000000001226

Lebedew, A. J., Dorman, J., Taylor, K.-L., Sheppard, J. M., Gabbett, T., \& Borgeaud, R. (2016). Development of a Repeated-Effort Test for Elite M en's Volleyball. International Journal of Sports Physiology and Performance, 2(3), 292-304. https:/ / doi. org/ 10.1123/ ijspp. 2.3.292

Markovic, G. (2007). Does plyometric training improve vertical jump height? A meta-analytical review. British Journal of Sports M edicine, 41(6), 349-355. https:/ / doi.org/ 10.1136/ bjsm.2007.035113

MARQUES, M.A.C.; GANZÁLEZ-BADILLO, J. J. (2006). 0 efeito do treino de força sobre 0 salto vertical em jogadores de basquetebol de 10-13 anos de idadeThe effects of strength training uponvertical jump in 10-13 years old basketball players. R. Bras Cl eM ov, 13(3), 51-58.

Martínez-rodríguez, A., Mira-alcaraz, J., Cuestas-calero, B. J., Pérez-turpín, J. A., \& Pedro, E. (2017). La Pliometría en el Voleibol Femenino. Revisión Sistemática. RETOS. NuevasTendencias En Educación Física, Deportey Recreación, 32, 208-213.

Martinez, A., Mira, J., \& Cuestas, B. (2017). La Pliometría en el Voleibol Femenino. Revisión Sistemática. / Plyometric Training in Female Volleyball Players. Systematic Review. Retos: N uevas Perspectivas de Educación Física, D eportey Recreación, 32, 208- 213. Retrieved from http:/ / search. ebscohost.com/

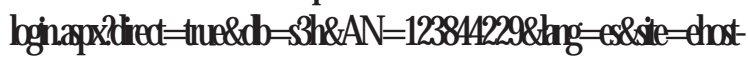
live\%0A http: / / content. ebscohost. com/

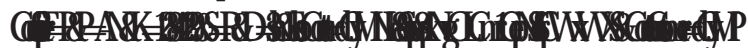

McK ay, H., Tsang, G., Heinonen, A., MacKelvie, K., Sanderson, D., \& K han, K. M. (2005). Ground reaction forces associated with an effective elementary school based jumping intervention. British Journal of Sports M edicine, 39(1), 10-14. https:/ / doi.org/ 10.1136/ bjsm. 2003.008615

Moran, J., Ramirez-Campillo, R., Liew, B., Chaabene, H., Behm, D. G., García-Hermoso, A., ... Granacher, U. (2020). Effects of Vertically and Horizontally O rientated Plyometric Training on Physical Performance: A Meta-analytical Comparison. Sports M edicine, (0123456789), 1-9. https:/ / doi.org/ 10.1007/ s40279-020-01340-6

Pereira, A., Costa, A. M., Santos, P., Figueiredo, T., \& João, P. V. (2015). Training strategy of explosive 
strength in young female volleyball players. M edicina (Kaunas, Lithuania), 51(2), 126-131. https:/ / doi.org/ 10.1016/ j.medici. 2015.03.004

Poulos, N., Chaouachi, A., Buchheit, M., Slimani, D., Haff, G. G. , N ewton, R. U. , \& Germain, P. S. (2018). Complex training and countermovement jump performance across multiple sets: Effect of back squat intensity. Kinesiology, 50, 75-89. https:/ / doi. org/ 10.1016/ j.geomorph.2007.03.005

Rafael M artin Acero, M iguel Fernández-del O Imo, Jose Andres Sánchez, X ose Luis 0 tero, Xavier Aguado, F. A. R. (2011). Reliability of Squat and Countermovement Jump Tests in Children 6 to 8 Years of Age. Pediatric Exercise Science, 23(1), 151160. https:/ / doi.org/ 10.1123/ pes.23.1. 151

Rajiæ Branislav, Dopsaj Milivoj , Carlos Pablos Abella, Vicente Caratalla Deval, K. S. (2013). Effects of combined and classic training on different isometric rate of force development parameters of leg extensors in female volleyball players: Discriminative analysis approach. Journal of Research in Medical Sciences, 18(10), 840-847.

Ramírez-Campillo, R., Gallardo, F., Henriquez-O lguín, C., Meylan, C. M. P., Martínez, C., Álvarez, C., ... Izquierdo, M. (2015). Effect of Vertical, Horizontal, and Combined Plyometric Training on Explosive, Balance, and Endurance Performance ofYoung Soccer Players. Journal of Strength and Conditioning Research, 29 (7), 1784-1795. https:/ / doi.org/ 10.1519/ JSC. 0000000000000827

Rodrigo Ramírez-Campillo, César Meylan, Cristian Alvarez, Carlos Henríquez-O Iguín, Cristian Martínez, Rodrigo Cañas-Jamett, David C Andrade, M. I. (2014). Effects of in-season low-volume highintensity plyometric training on explosive actions and endurance of young soccer players. Journal of Strength and Conditioning Research, 28(5), 1335- 1342. https:/ / doi.org/ 10.1519/ JSC.0000000000000284

Saez deVillarreal, E, Suarez-Arrones, L, Requena, B, Haff, GG, Ferrete, C. (2015). Effects of plyometric and sprint training on physical and technical skill performance in adolescent soccer players. Journal of Strength \& Conditioning Research, 29(7), 1894- 1903.

Sánchez-Moreno, M., García-A sencio, C., GonzálezBadillo, J. J., \& Díaz-Cueli, D. (2018). Strength and vertical jump performance changes in elite male volleyball players during the season. Retos, 2041(34), 291-294.

Sánchez-Sixto, A., \& Floría, P. (2016). Efecto del entrenamiento combinado de fuerza y pliometría en va riables biomecánicas del salto vertical en jugadoras de baloncesto. Retos, (31), 114- 117.

Sattler, T., Idrizovic, K. , João, P.V., Spasic, M., Gjinovci, B., Uljevic, 0., \& Sekulic, D. (2018). The Effects of 3-Month Skill-Based and Plyometric Conditioning on Fitness Parameters in Junior Female Volleyball Players. Pediatric Exercise Science, 30(3), 353- 363. https:/ / doi.org/ 10.1123/ pes. 2017-0178

Seitz, L. B., \& Haff, G. G. (2016). Factors Modulating Post-Activation Potentiation of J ump, Sprint, Throw, and U pper-Body Ballistic Performances: A Systematic Review with Meta-Analysis. Sports M edicine, 46(2), 231-240. https:/ / doi. org/ 10.1007/ s40279-0150415-7

Soylu, C.., Altundað, E., Akarçe-me, C., \&Yildirim, N. Ü. (2020). The relationship between isokinetic knee flexion and extension muscle strength, jump performance, dynamic balance and injury risk in female volleyball players. Journal of Human Sport and Exercise, 15(3), 502-514. https:/ / doi.org/ 10.14198/ jhse. 2020.153.03

Stojanoviæ, E. , Ristiæ, V., M cM aster, D.T. , \& M ilanoviæ, Z. (2017). Effect of Plyometric Training on Vertical Jump Performance in FemaleAthletes: A Systematic Review and Meta-Analysis. Sports Medicine, 47(5), 975-986. https:/ / doi. org/ 10.1007/ s40279-0160634-6

Tillin, N. A., Pain, M. T. G., \& Folland, J. P. (2013). Identification of contraction onset during explosive contractions. Response to Thompson et al. «Consistency of rapid muscle force characteristics: Influence of muscle contraction onset detection methodology» [] Electromyogr Kinesiol 2012;22(6):893-900]. Journal of Electromyography and Kinesiology, 23(4), 991-994. https:/ / doi. org/ 10.1016/ j.jelekin.2013.04.015

VILELA, G; Da SILVA, S. F. (2017). Efeitos do treinamento pliométrico na força explosiva e potência de meninas púberes praticantes de voleibol. Revista Brasilei ra Ciência eM ovimento, 25(1), 109-117. Ziv, G., \& Lidor, R. (2010). Vertical jump in female and male volleyball players: a review of observational and experimental studies. Scandinavian Journal of $\mathrm{Me}$ dicine \& Science in Sports, 20 (4), 556-567. https:/ / doi.org/ 10.1111/ j.1600-0838.2009.01083.x 